\title{
Damage localization of closing cracks using a signal decomposition technique
}

\author{
J. Prawin, A. Rama Mohan Rao \\ CSIR Structural Engineering Research Centre, India \\ prawinpsg@gmail.com, bttp:// orcid.org/0000-0002-7579-025X \\ arm2956@yahoo.com, bttp://orcid.org/0000-0002-6405-3633
}

\begin{abstract}
Fatigue cracks are a common occurrence in engineering structures subjected to dynamic loading and need to identify at its earliest stage before it leads to catastrophic failure. The presence of fatigue-breathing crack or closing cracks is usually characterised by the presence of sub and superharmonics in the response of the structure subjected to harmonic excitation. It should be mentioned here that the amplitude of nonlinear harmonics are of very less order in magnitude when compared to linear or excitation component. Further, these nonlinear components often get buried in noise as both are having matched (low) energy levels. The present work attempts to decompose the acceleration time history response using singular spectrum analysis and propose a strategy to extract the nonlinear components from the residual noisy time history component. A new damage index based on these extracted nonlinear features is also proposed for closing crack localization. The effectiveness of the proposed closing crack localization approach is illustrated using detailed numerical studies and validated with lab level experimentation on the simple beam-like structure. It can be concluded from the investigations that the proposed signal decomposition based damage localization technique can detect and locate more than one crack present in the structure.
\end{abstract}

KEYWORDS. Pairwise eigenvalues; Singular spectrum analysis; Closing crack; Damage localization; Nonlinearity; Bilinear.

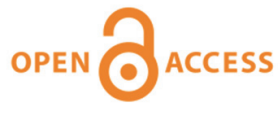

Citation: Prawin, J., Rama Mohan Rao, A., Damage Localization of closing cracks using a signal decomposition technique, Frattura ed Integrità Strutturale, 48 (2019) 513-522.

Received: 15.11 .2018

Accepted: 22.02 .2019

Published: 01.04.2019

Copyright: (C) 2019 This is an open access article under the terms of the CC-BY 4.0, which permits unrestricted use, distribution, and reproduction in any medium, provided the original author and source are credited.

\section{INTRODUCTION}

S ingular Spectrum Analysis (SSA) is a popular multivariate analysis technique widely used for signal decomposition and signal approximation. SSA essentially can be described in four steps namely embedding, Singular Value Decomposition (SVD), grouping and diagonal averaging. The Complete description of the technique can be found in Golyandina et al., [1].

SSA is popularly used in several diverse areas like climate change, geophysical phenomena, mineral processing, seismic data processing, and telecommunication applications [1-3]. SSA has been recently applied in the area of structural health 
monitoring and found to be very effective. A detailed review of earlier works of SSA on structural health monitoring applications can be found in Prawin et.al, [4]. In most of the earlier works on SSA in SHM applications, the noisy components are neglected during the reconstruction and transformation back to the actual response. Oliveria et al., [5] recently reported that the discontinuity related damage sensitive features are found in these ignored residual noise components of the actual response. In view of this, we process further the residual (noisy) components obtained from SSA to reliably check whether any higher order or superharmonic resonances present or buried in the residual signal. Further, the proposed SSA based decomposition technique can reliably extract all the higher order harmonic components in contrast to the existing approaches which are based on only first few harmonics and the damage detection can be enhanced with consideration of more number of super harmonics.

\section{SIGNAL DECOMPOSITION USING SINGULAR SPECTRUM ANALYSIS}

7 he breathing crack is usually assessed by the spectrum of the response of the structure measured at a particular location. The presence of super harmonics (i.e. higher order harmonics) apart from the fundamental excitation harmonic confirms the nonlinear behaviour induced by closing crack when the cracked structure is subjected to harmonic excitation. Earlier researchers [6-8] basically employed the ratio of the power spectrum amplitude of the response at second or third order harmonic to the first order/linear/excitation harmonic as damage index for closing crack localization. Few other researchers [6-8] have used the spatial curvature of the ratio of the power spectrum of superharmonics to excitation harmonic as damage index due to the fact that the second spatial derivative magnifies the cracks/discontinuity in the response. Further, most of the existing techniques consider only one or two higher order harmonic frequencies for closing crack localization and the rich damage sensitive features present in the higher order harmonics have been ignored. The basic reason behind this is that the amplitude of super harmonics (i.e. nonlinear harmonics) are of very less order in magnitude when compared to linear fundamental excitation harmonic. These higher order nonlinear harmonic components also often get buried in noise and difficult to conclude whether it is noise or nonlinear component as both are having matched (low) energy levels. Therefore, the extraction of these harmonics under noisy environment and damage detection at its incipient stage is highly challenging.

In the proposed closing crack localization technique, the cracked structure i.e. with closing crack is subjected to harmonic excitation with a particular single frequency; therefore the response is also harmonic in nature. As mentioned earlier, the response of the cracked structure, i.e. with closing crack vibrates at the excitation frequency as well as the higher order super harmonics of the excitation frequency. In contrast, the healthy structure (i.e., without breathing crack) vibrates only at excitation frequency due to single tone harmonic excitation. Since the response of the structure is harmonic in nature, the decomposition of the time history data of the cracked structure, i.e., structural or structural component with closing/breathing crack at a particular location, by SSA contains only harmonic components (i.e. both linear excitation and nonlinear super harmonic harmonics) and noise. Harmonic signals basically exhibit two eigen triples with close singular values [1-5]. Therefore, this property helps in easy interpretation and identification of the harmonic signal components during decomposition of the measured response by SSA. The number of pairwise eigenvalues indicates the number of harmonic components present in the response. Since the structure is excited by a single tone harmonic load, there exists only one pairwise eigenvalue for the structure without a closing crack. In contrast, there will be more than one pairwise eigenvalues exist for the structure with closing crack due to the presence of super harmonics. These harmonic components (i.e. pairwise singular values) are present in descending order (i.e. decreasing) of their energy corresponding to each frequency due to the property of SSA. The first pairwise eigenvalue will be always the linear excitation harmonic component and it always exhibits dominant energy for both healthy and cracked structure (i.e. structure with breathing crack). This can be easily verified by the Fourier power spectrum of the respective harmonic component. The subsequent pairwise singular values (i.e. present with low energy levels) represent the nonlinear superharmonic components. The contribution of higher energy in the case of fundamental excitation harmonic is clearly justified by the fact that the amplitude of fundamental harmonic is of two or three order higher in magnitude when compared to the amplitude of nonlinear superharmonic. In the present work, the linear components can be isolated by the first pairwise singular value. It should be mentioned here even though there is no theoretical evidence, extensive studies carried out on closing crack problems reveal that the energy content of the dominant pairwise eigenvalues corresponding to linear response constitutes $99 \%$ of the total energy $[4,7,9]$. However, to be on the safer side, we prefer to use the first pairwise singular value for extracting the linear fundamental excitation harmonic component.

In order to reliably extract the low energy nonlinear harmonics, we apply SSA again on the residual (noisy) components obtained from SSA after ignoring linear harmonic components. This is in contrast to the earlier works [1-3] where the noisy 
components are usually neglected during the reconstruction of the response. SSA has been performed repeatedly twice on the response in the proposed closing crack localization technique, to isolate the linear components from the actual response in the first instance and to identify the presence or absence of the higher order harmonic resonances from the residual/noisy part of the signal in the second instance. It should be mentioned here that the residual components contain the nonlinear components. The higher order harmonic components, induced by the nonlinear behaviour of the closing crack, are usually buried in the residual component and it is easily detected by the same pairwise eigenvalue concept mentioned earlier in the proposed technique. This clearly demonstrates the ability of the SSA in decomposing the linear, nonlinear harmonic and noise components from the total response.

\section{Damage diagnostic scheme based on singular spectrum analysis}

The steps involved in closing crack localization based on the signal decomposition technique are

1. Decompose the acceleration time history response obtained at various spatial locations across the structure using SSA.

2. The linear and nonlinear harmonic components are identified through pairwise eigenvalues concept. The first pair indicates the linear component and the rest are nonlinear harmonic components generated by the closing crack. However, these nonlinear harmonics are buried in the noisy components and difficult to extract reliably at this stage

3. Reconstruct the residual components (eliminating first Eigen pair component) by grouping and diagonal averaging of SSA technique.

4. Decompose the residual time history response obtained from step-3 using SSA. The nonlinear components are identified by pairwise eigenvalue concept.

5. The cumulative sum of the peak amplitudes of each of the nonlinear harmonic component is considered as damage index. The damage index is defined as follows

$$
D I(i)=\sum_{k=1}^{n f} \mid A_{\text {peak, }, k}(\text { sup erbarmonic nonlinear component }) \mid
$$

where DI (i) indicates the damage index of the $i^{\text {th }}$ degree of freedom (or sensor node) and nf, $A_{\text {peak, }}$ represent the number of superharmonic frequencies and the peak Fourier power spectrum amplitude of the selected ' $\mathrm{k}$ 'th nonlinear harmonic component. The damage index is computed for each sensor location. The sensor node exhibiting the higher magnitude of magnitude index is the true spatial location of the breathing crack

\section{NUMERICAL INVESTIGATIONS}

A simple beam like structures such as simply supported beam and cantilever beam, simulated with closing cracks at varied spatial locations are chosen as numerical examples; to test the SSA based closing crack localization technique. Since we have also carried out experimental investigations using the cantilever beam, and the numerical investigations carried out on both the beams are similar in nature, we present only the results of the simply supported beam in this paper to avoid repetition or redundancy. However, the experimental studies on the cantilever beam are presented later in this paper. Steel cracked simply supported beam given in Fig. 1 is chosen in the present work for numerical investigation. The span of the beam is $0.7 \mathrm{~m}$ and has an area of $4 \mathrm{e}-4 \mathrm{~m} 2$ and Moment of Inertia as $0.667 \mathrm{e}-8 \mathrm{~m} 4$. The natural frequencies of the underlying linear healthy beam are found to be $89.671 \mathrm{~Hz}, 354.689 \mathrm{~Hz}, 799.16 \mathrm{~Hz}, 1419.46 \mathrm{~Hz}, 1711.46 \mathrm{~Hz}$ and $2218.17 \mathrm{~Hz}$. The finite element model of the beam considers standard one-dimensional Euler-Bernoulli beam elements with two nodes per beam element and each node have three degrees of freedom; longitudinal displacement, translational displacement and bending rotation. Heavy side step function, widely preferred by the researchers is used in the present work to model the opening-closing behaviour of the breathing crack. The damaged element stiffness matrix with the bilinear behaviour induced by the rotations $\left(\theta_{i}, \theta_{j}\right)$ at the nodes of the respective damaged element [6-9] is given by

$$
K_{d}=K-H\left(\theta_{i}-\theta_{j}\right) K_{c}:\left\{\begin{array}{l}
H\left(\theta_{i}-\theta_{j}\right)=1, \theta_{i}>\theta_{j} \\
H\left(\theta_{i}-\theta_{j}\right)=0, \theta_{i}<\theta_{j}
\end{array}\right.
$$


where $\mathrm{H}, \mathrm{K}, \mathrm{K}_{\mathrm{d}}, \mathrm{K}_{\mathrm{c}}$ indicates the Heaviside step function, undamaged, damaged (breathing crack) and cracked (open) stiffness of a particular element connected by nodes ' $i$ ' and ' $j$ '. The crack depth is defined in the cracked stiffness matrix $K_{c}$. The detailed formulations are deliberately omitted here as they are of least consequence.

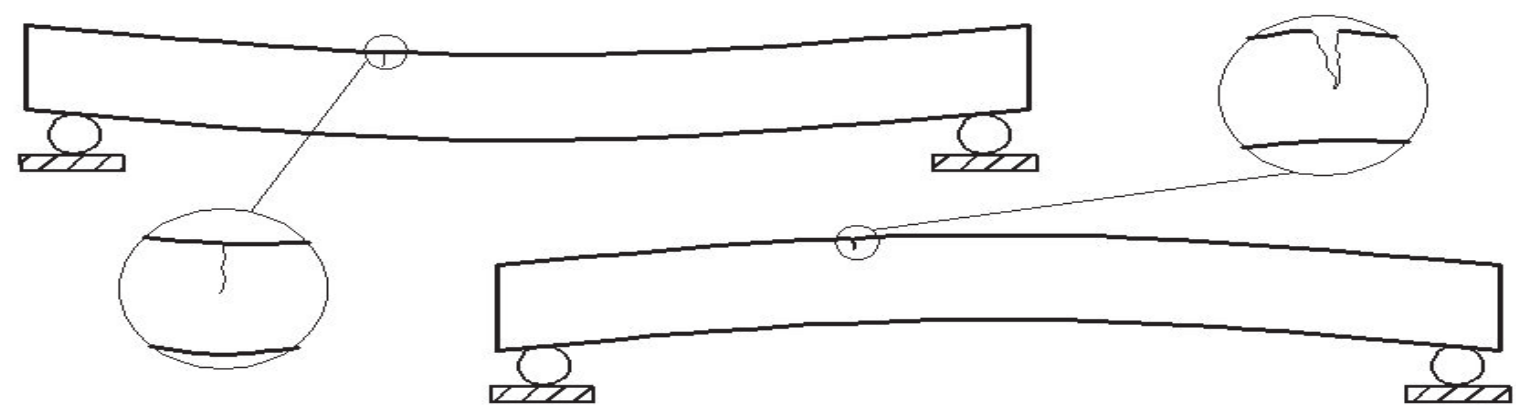

Figure 1: Simply supported beam with a closing crack.

The beam is discretised with 10 elements. The closing crack is simulated near $2 / 3$ span of the simply supported beam from the left end i.e. element no. 7 with the crack depth of about $7 \%$ of the total depth. The beam is excited at the centre with an excitation frequency of $90 \mathrm{~Hz}$. The acceleration time history responses are obtained at nine locations (i.e. finite element model discretised with 10 elements) spatially across the beam with a spacing of $0.07 \mathrm{~m}$ per element. The obtained acceleration time history spatially across the beam is polluted with $10 \%$ standard white Gaussian noise (i.e. SNR $=30$ ) before postprocessing to test the applicability of the proposed signal decomposition technique in the presence of noise. However, investigations have also been carried out without measurement noise for comparison purposes.

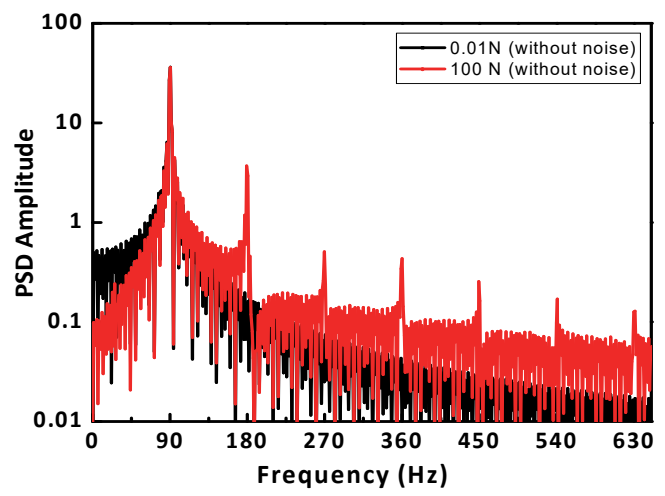

(a)

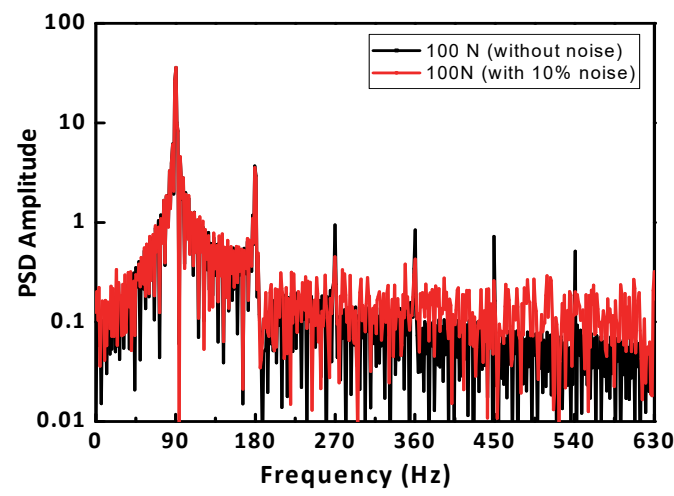

(b)

Figure 2: Fourier Power Spectrum (a) undamaged and damaged data without noise (b) damaged data with and without noise

The acceleration time history responses are obtained from the cracked structure at two different excitation amplitudes of $0.01 \mathrm{~N}$ and $100 \mathrm{~N}$. The spectral density plot of the cracked structure obtained under these two different excitation amplitudes are shown in Fig. 2(a). The spectral density plot of the cracked structure using noise-free and noisy measurements under $100 \mathrm{~N}$ excitation is presented in Fig. 2(b). The spectral density plot corresponding to $0.01 \mathrm{~N}$, presented in Fig. 2(a) shows a single peak at $90 \mathrm{~Hz}$. This confirms that the cracked structure behaves linearly under $0.01 \mathrm{~N}$ excitation as the structure vibrates only at its excitation frequency. The spectral density plot corresponding to $100 \mathrm{~N}$ excitation with noise-free measurements, in contrast to $0.01 \mathrm{~N}$ excitation vibrates not only at its excitation frequency i.e. $90 \mathrm{~Hz}$ but also at its super harmonics, $90 \mathrm{~Hz}, 180 \mathrm{~Hz}, 270 \mathrm{~Hz}, 360 \mathrm{~Hz}$, and so on. This is evident from the peaks at those frequencies in Fig. 2 (a). It can be observed from Fig. 2(a) that the peak at fundamental excitation harmonic exhibit very high magnitude when compared to the magnitude of the peaks at their respective super harmonics. The presence of additional superharmonics apart from the fundamental excitation harmonic concludes the nonlinear behaviour induced by the closing cracks. It can be observed from Fig. 2(b) that the spectral density plot of the cracked structure corresponding to $100 \mathrm{~N}$ excitation with noisy measurements shows a peak at the excitation frequency and its superharmonics. However, the peak at first superharmonic, i.e. $180 \mathrm{~Hz}$ is only clearly visible, while the higher order superharmonics get buried in the noise as both exhibit similar energy 
levels. It should be mentioned here that using the conventional spectral analysis, only one or two superharmonics can be extracted reliably under noisy environment while using the proposed approach (SSA), a large number of superharmonics are extractable.

In the present work, for the application of SSA on the time history response, the window length is selected as the time lag corresponding to the first zero crossing between L/4 and L/2, computed based on autocorrelation [4]. More details related to the choice of window length are not discussed here, as it deviates from the scope of the present work. However, details on the choice of the window length can be found in Prawin et.al. [4].

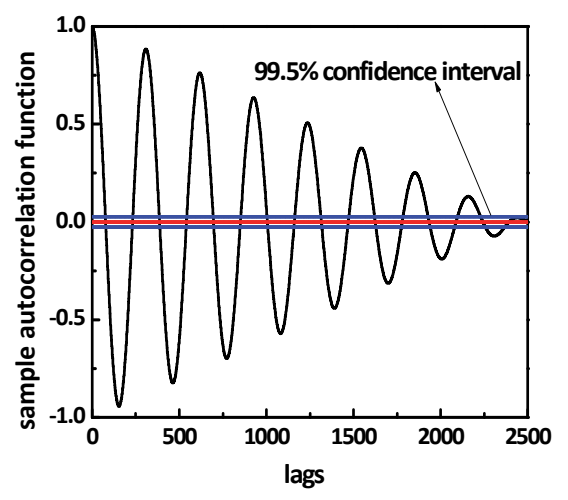

(a)

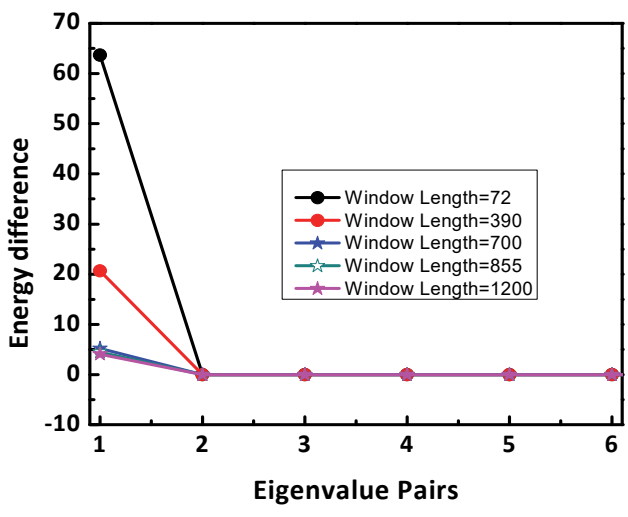

(b)

Figure 3: (a) Autocorrelation function (b) Window Length

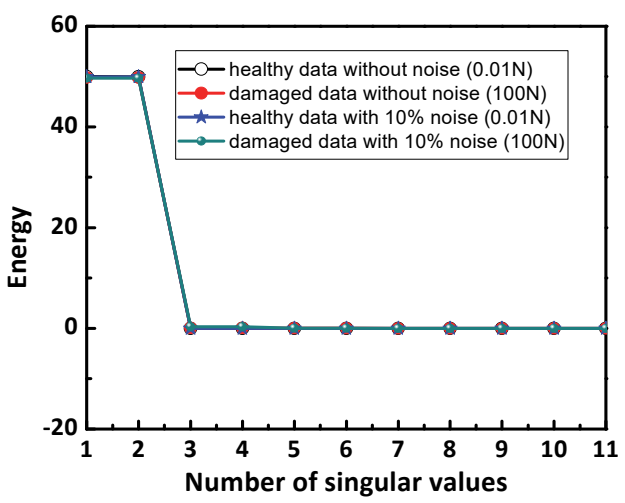

(a)

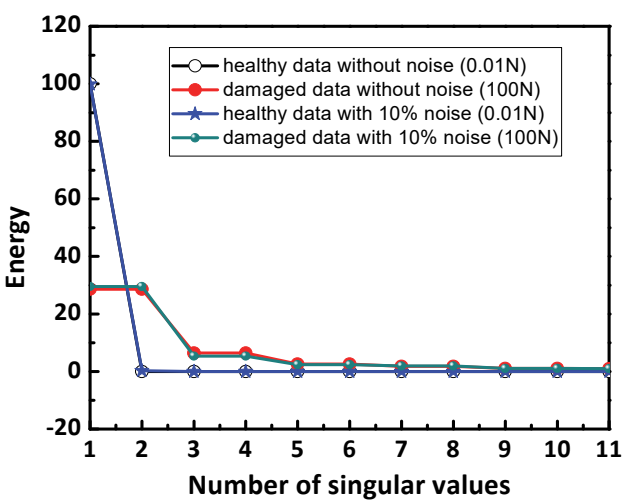

(b)

Figure 4: Singular Spectrum- (a) actual (b) residual acceleration time history

Fig. 3 (a) depicts the autocorrelation of the response measured at $0.4 \mathrm{~m}$ from the left support corresponding to $100 \mathrm{~N}$ excitation. Fig. 3(a) shows the zero crossing of the autocorrelation function of the response at time lags around 72, 232, 387, 545, 698, 851, 1008, 1196, 1315, 1470, and 1625 and so on. Fig. 3(b) shows the plot corresponding to the energy difference between eigenpairs with varied window lengths chosen based on the time lags corresponding to zero crossing of the response. The energy difference (of eigenpairs) plot furnished in Fig. 3(b) shows zero magnitudes when the length of the window is considered as 1190 (or above). Based on this investigation, the above-automated choice of window length is chosen in the present work. Once the window length is chosen, the next stage of SSA can be performed on the response to localize the closing crack.

The singular spectrum of the noise-free response and response polluted with $10 \%$ noise (i.e. node 5) obtained by SSA is given in Fig. 4 (a), while the results of the residual response is given in Fig. 4 (b). Both Figs. 4 (a) and 4 (b) furnish the results corresponding to two different excitation amplitudes of $0.01 \mathrm{~N}$ and $100 \mathrm{~N}$. Fig. 4(a) concludes that the eigentriples with close singular values exist for the total actual response corresponding to both excitation amplitudes of $0.01 \mathrm{~N}$ and $1 \mathrm{~N}$. While the pairwise component i.e. harmonic signal is absent in Fig. 4 (b) for the low amplitude of excitation and present for the high amplitude of excitation even with the noisy measurements. Each of the pairwise singular values in the residual signal of $100 \mathrm{~N}$ excitation corresponds to nonlinear harmonics components i.e. super harmonic components generated due to the 
closing crack in the structure. The nonlinear components are isolated using SSA and damage index is then computed for closing crack localization.

\section{Closing Crack Localization - two different test cases}

The simply supported beam is simulated with closing crack at two different spatial locations in order to illustrate the robustness of the proposed technique in localizing the closing crack present anywhere in the structure. The first test case considers the breathing crack located at element no, 4, while the second test case considers the breathing crack at element no.7. Both the test cases have the same crack depth equal to $7 \%$ of the total depth. The results of the damage index evaluated for these two varied crack locations is shown in Fig. 5 using the noise-free and noisy time history response (i.e. actual response polluted with 10\% noise before processing) measured at varied locations spatially across the structure. It can be concluded from Fig. 5 that the maximum value of the damage index in both the test cases considered is at the damaged element of the beam even with noisy measurements.

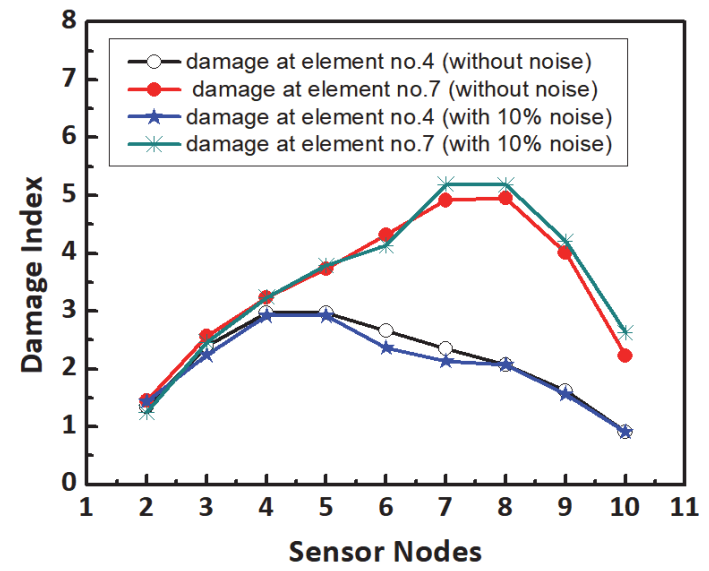

Figure 5: Damage index based on SSA - 9 sensors.

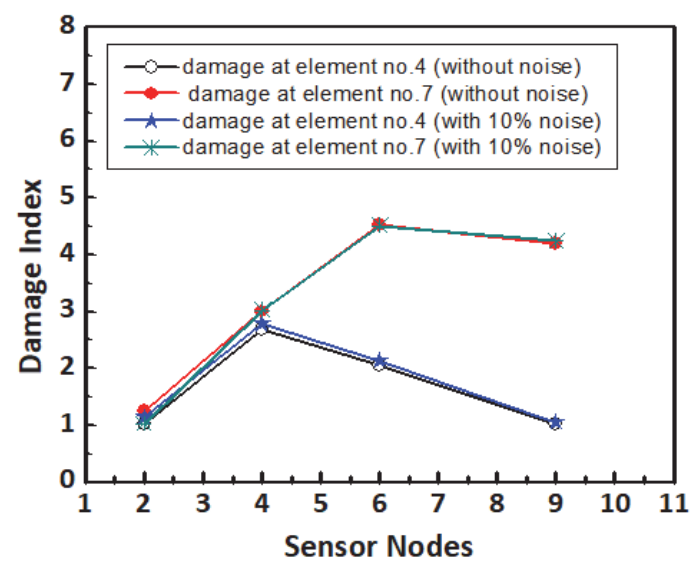

Figure 6: Damage index based on SSA - 4 sensors.

\section{Closing Crack Localization with Limited instrumentation}

In order to investigate the effectiveness of the proposed closing crack localization approach with limited sensors, we have used the measurements obtained at 4 selective locations (i.e. at nodes 2, 4, 6, 9), identified using the popular Effective Independence optimal sensor placement technique [10]. The results of the damage index, obtained with limited measurements for the above damage cases of Fig. 5 is presented in Fig. 6. It can be observed from Fig. 6 that the maximum value of the damage index is at node no.4 for the case of damage simulated in element no.4. The maximum value of the damage index for the case of damage simulated in element no. 7 is at node no.6, which is close to the nodes 7-8 (i.e. element no. 7). Therefore, with limited instrumentation, the closest possible spatial location of damage can be identified, while with more sensors, the maximum value of damage will be occurring exactly between the two closely spaced nodes corresponding to damaged element (i.e. peak at nodes 7-8 in the case of damage at element no. 7, as evident from Fig. 5). This investigation clearly concludes that the proposed algorithm has the ability to localize the closing crack even with limited measurements.

\section{Comparison with Previous work}

In order to demonstrate the effectiveness of the proposed algorithm in identifying smaller and subtle cracks, the results of the proposed damage index are compared with the previous vibration based breathing crack identification techniques based on only first few super harmonics [6-8]. The various damage indices given in the reported relevant research work in the literature are summarized as follows

$$
\begin{aligned}
& \left(D I_{1}\right)_{i}=\frac{\sum_{j=1}^{n} A_{j \omega_{X}}^{i}}{A_{\omega_{X}}^{i}} ; \mathrm{i}=1,2,3, \ldots \mathrm{N} ; \\
& \left(D I_{2}\right)_{i}=d^{2}\left(\left(D I_{1}\right)_{i}\right) ; \mathrm{i}=2,3, \ldots \mathrm{N}-1 ; d^{2}\left(\left(D I_{1}\right)_{i}\right)=\frac{\left(D I_{1}\right)_{i+1}-2\left(D I_{1}\right)_{i}+\left(D I_{1}\right)_{i-1}}{b^{2}}
\end{aligned}
$$


where the first damage index $\left(D I_{1}\right)_{i}$ indicates the ratio of the sum of the power spectrum amplitudes of ' $n$ ' superharmonics to power spectrum amplitude of linear excitation harmonic (i.e FFT amplitude (A) at excitation frequency $\omega_{X}$ ). The second damage index indicates the extension of the first approach to the curvature based index and $\mathrm{N}$ represents the total number of degrees of freedom.

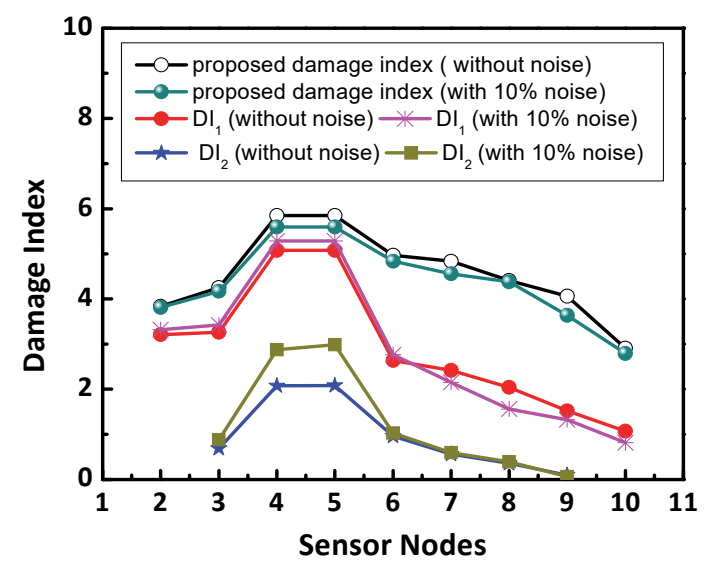

(a)

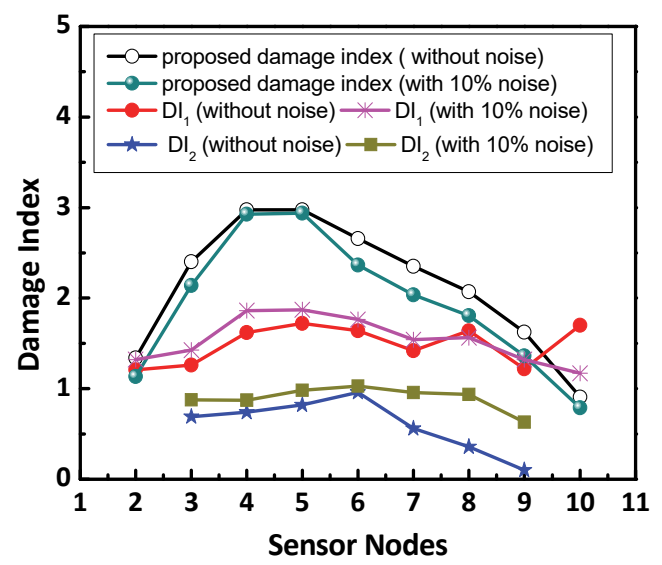

(b)

Figure 7: Various damage indices (a) 20\% crack depth (b) 7\% crack depth

For this investigation on the proposed damage index with previous works, the closing crack is simulated in simply supported beam in element no. 4 with crack depth equal to $20 \%$ and $7 \%$ of overall depth of the beam. The results of the damage index estimated using Eqn. (1) and Eqn. (3) is furnished in Fig. 7 (a) for the beam having crack equal to $20 \%$ of total depth. Similarly the results of the damage index corresponding to the case of simply supported beam with crack depth equal to $7 \%$ of overall depth of the beam is shown in Fig. 7 (b). Before computing the damage index, the computed acceleration time history responses are polluted with 10\% noise. It can be observed from Fig. 7 (a) that the peak value of all the damage indices is at the exact location of the breathing crack. However it can be observed from Fig. 7 (b), the two damage indices corresponding to the previous approaches fail to detect smaller cracks due to consideration of only a few superharmonics and difficulty in distinguishing noise and true nonlinear harmonic. For example, the damage index $D I_{1}$ shows multiple peaks which creates confusion and difficult to conclude the exact location of the breathing crack. While the damage index $\mathrm{DI}_{2}$ shows a peak at the wrong location. Therefore, the earlier approaches [6-8] fail to detect subtle cracks; however, the proposed method identifies the minor damages very precisely by reliably extracting all the possible higher order harmonics.

\section{EXPERIMENTAL VALIDATION}

A part from the above numerical investigations, experimental studies have been carried out by considering a cantilever beam with single and multiple breathing cracks (i.e. two test specimens), to test and verify the proposed closing crack localization algorithm. The experimental set up followed in the present work is popularly used by Prime et.al. [11] and Douka et.al., [12] earlier for validation of their crack diagnosis algorithms. The single crack test beam shown in Fig. 8 , is constructed by bonding two aluminium alloy beams (i.e. four pieces) together. The faces of the top plates in contact induce breathing behaviour. The instrumentation set up is same for both the specimens and shown in Fig. 8 . The length of the beam is $1 \mathrm{~m}$ for both the test specimens. The cross-section dimension of both the specimens is same and found to be $0.0254 \times 0.0127 \mathrm{~m}$. The ratio of the thickness of the top and bottom plates decides the crack depth. The top and bottom plate thicknesses corresponding to single crack test specimen (case-1) are $1.5875 \mathrm{~mm}$ and $11.1125 \mathrm{~mm}$ respectively. This results in a crack depth of $12.5 \%$ of the overall depth of the beam and the crack is simulated at $0.4 \mathrm{~m}$ (i.e. located between sensor 3 and 4) from the fixed end. Similarly, the top and bottom plate thicknesses corresponding to two crack specimen (case-2) are $3.175 \mathrm{~mm}$ and $9.525 \mathrm{~mm}$ which results in $25 \%$ crack depth of the total depth of the beam. In the case of two crack experimental specimen shown in Fig. 9, the first crack at $0.2 \mathrm{~m}$ is located between sensor 2 and 3 (closer to sensor 2), while the second crack at $0.7 \mathrm{~m}$ from the fixed end is located between sensor 5 and 6 (closer to sensor 6 ). The two crack 
experimental specimen, employed in the present work was earlier used by the authors for validating their proposed damage diagnosis algorithm [4].

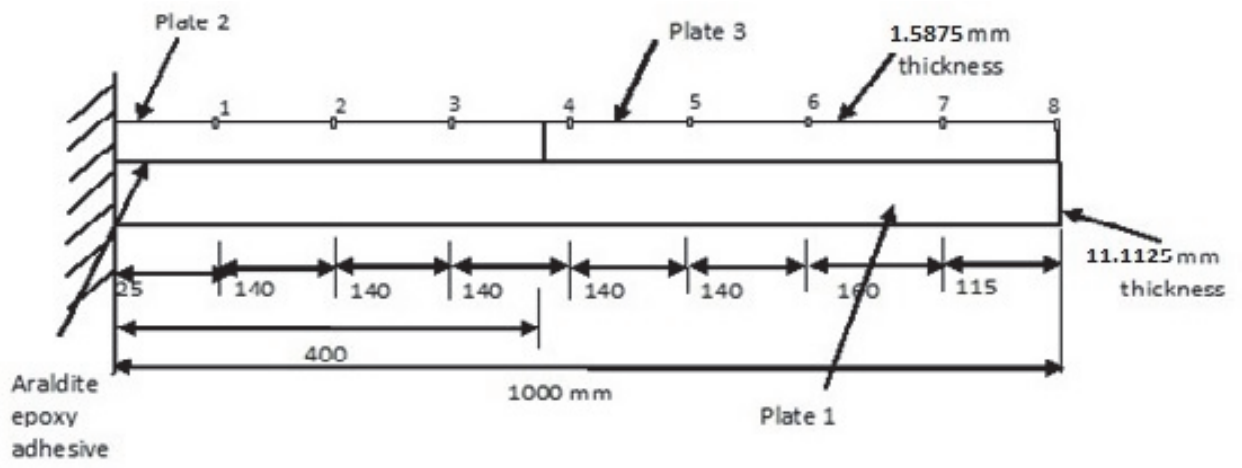

Figure 8: Instrumentation set up - Single Crack Crack specimen

Both the experimental specimens are excited near the centre of the beam with harmonic excitation of $10 \mathrm{~Hz}$. The acceleration time history responses are measured at the eight spatial locations as indicated in Fig. 8. The damage index based on SSA is estimated using Eqn. (1) for both the test specimens and the results are furnished in Fig. 10. The damage index corresponding to single crack experimental specimen shows a single peak at sensor 4, which coincides with the exact location of the actual crack. It is also evident from Fig. 10 that the damage index plot corresponding to two crack experimental specimen shows peaks at the spatial location of sensor 3 and sensor 6 clearly reflect the actual closing crack locations of the considered beam.

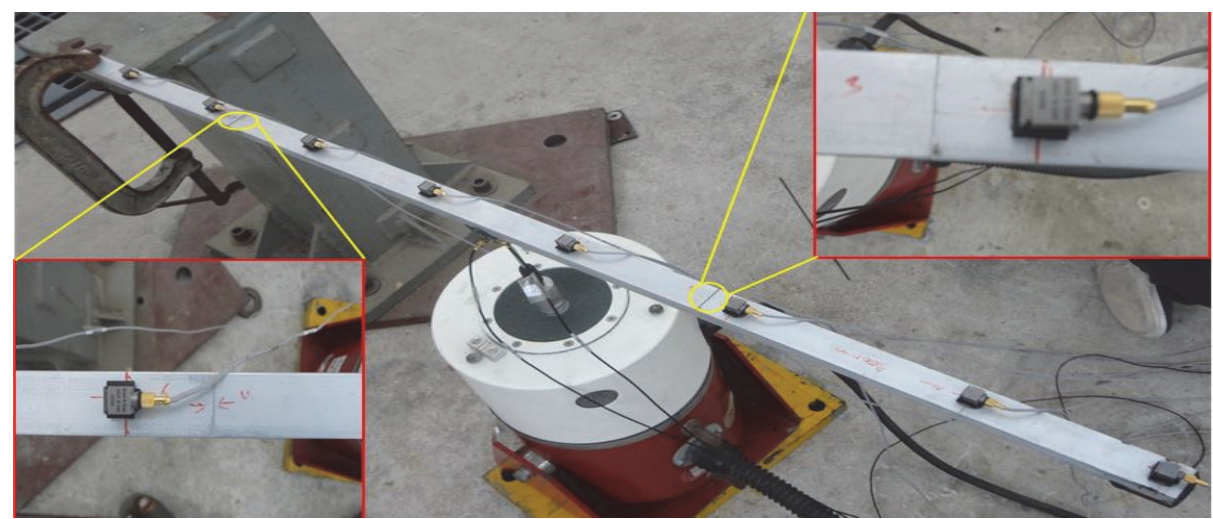

Figure 9: Experimental Multiple Breathing Crack specimen [4]

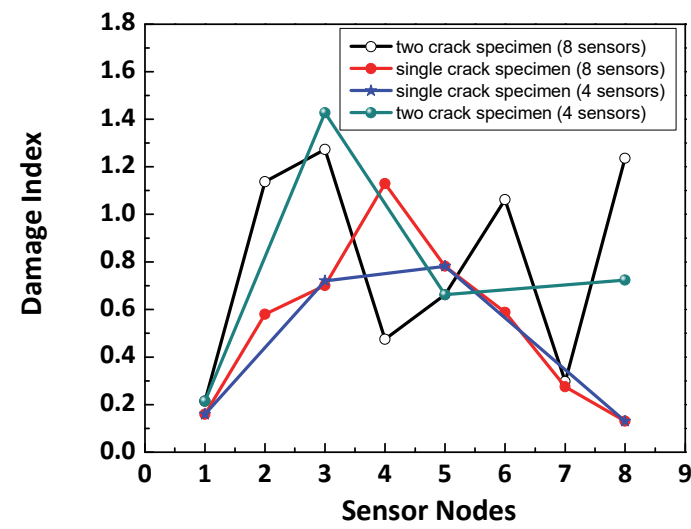

Figure 10: Damage Index - Experimental Specimen - multiple breathing cracks 
In order to test the robustness of the proposed algorithm with limited measurements experimentally, the measurements of both the single and two crack specimens at optimally chosen four sensors i.e., at nodes 1, 3, 5 and 8 are only considered instead of all the 8 sensors. The damage index is computed using the limited sensor information. The corresponding results are also presented in Fig. 10. It can be observed from Fig. 10 that the damage index exhibit pairwise peaks at nodes 3 and 5 for the single crack specimen where the crack is actually located close to the sensor node 4 . It can also be concluded from Fig. 10 that in the case of two-crack specimen, only one crack at sensor node 3 (i.e. the crack is actually located between nodes 2 and 3 ) is detectable, with limited measurements of 4 sensors. This study clearly illustrate that only he possible region of spatial location of closing crack can be identified with limited instrumentation and it is also difficult to localize multiple cracks. From the numerical and experimental investigations, it is clear that the proposed signal decomposition approach based on singular spectrum analysis can identify more than one cracks present anywhere in the structure and of any crack depth with minimum optimal number of sensors.

\section{CONCLUSIONS}

A signal decomposition based technique has been proposed in this paper to identify the smaller and subtle closing cracks present anywhere in the structure. Both experimental and numerical investigations have been carried out on simple beam-like structures to test the robustness and effectiveness of the proposed signal decomposition based breathing crack localization technique. The results furnished from the investigations are given below

1. The proposed signal decomposition is capable of isolating linear, nonlinear and noise components effectively.

2. The proposed SSA based damage diagnostic technique is robust enough to locate even more than one crack present in the structure.

3. Numerical investigations conclude that the proposed breathing crack localization technique is reference free and can detect minor crack of about $7 \%$ crack depth. Experimental investigations conclude that the proposed technique can detect crack depth of about $12.5 \%$ of overall depth of the beam.

4. The proposed approach can identify the breathing crack even with the acceleration responses being polluted with $10 \%$ measurement noise.

5. The proposed closing crack localization technique can also identify smaller cracks due to consideration of more number of super harmonics in contrast to the earlier works which consider only first few super harmonics.

\section{ACKNOWLEDGEMENTS}

he authors thank the technical staff of ASTAR and SHML Lab of CSIR-SERC for their support during laboratory testing.

\section{REFERENCES}

[1] Golyandina, N., Nekrutkin, V. and Zhigljavsky, A.A. (2001). Analysis of time series structure: SSA and related techniques. Chapman and Hall/CRC.

[2] Vautard, R., Yiou, P. and Ghil, M. (1992). Singular-spectrum analysis: A toolkit for short, noisy chaotic signals. Physica D: Nonlinear Phenomena, 58(1-4), pp.95-126.

[3] Loh, C.H., Chen, C.H. and Hsu, T.Y. (2011). Application of advanced statistical methods for extracting long-term trends in static monitoring data from an arch dam. Structural Health Monitoring, 10(6), pp.587-601.

[4] Prawin, J., Lakshmi, K. and Rao, A.R.M. (2018). A novel singular spectrum analysis-based baseline-free approach for fatigue-breathing crack identification. Journal of Intelligent Material Systems and Structures, 29(10), pp.2249-2266.

[5] De Oliveira, M.A., Vieira Filho, J., Lopes Jr, V. and Inman, D.J. (2017). A new approach for structural damage detection exploring the singular spectrum analysis. Journal of Intelligent Material Systems and Structures, 28(9), pp.1160-1174.

[6] Shen, M.H. and Chu, Y.C. (1992). Vibrations of beams with a fatigue crack. Computers \& Structures, 45(1), pp.79-93. 
[7] Giannini, O., Casini, P. and Vestroni, F. (2013). Nonlinear harmonic identification of breathing cracks in beams. Computers \& Structures, 129, pp.166-177.

[8] Bovsunovsky, A. and Surace, C. (2015). Non-linearities in the vibrations of elastic structures with a closing crack: a state of the art review. Mechanical Systems and Signal Processing, 62, pp.129-148.

[9] Prawin, J., Rao, A.R.M. and Lakshmi, K. (2015). Nonlinear identification of structures using ambient vibration data. Computers \& Structures, 154, pp.116-134.

[10] Kammer, D.C. (2005). Sensor set expansion for modal vibration testing. Mechanical systems and signal processing, 19(4), pp.700-713.

[11] Prime, M.B. and Shevitz, D.W. (1995). Linear and nonlinear methods for detecting cracks in beams (No. LA-UR-954008; CONF-960238-10). Los Alamos National Lab., NM (United States).

[12] Douka, E. and Hadjileontiadis, L.J. (2005). Time-frequency analysis of the free vibration response of a beam with a breathing crack. Ndt \& E International, 38(1), pp.3-10. 